\title{
Analyzing the Spread of COVID-19 Disease Using An Age-Structured Model: Application to Italy, Spain, France, UK, and Algeria Using Early Data
}

\section{Salih Djilali}

University of Algereia

Sunil Kumar ( $\nabla$ skumar.math@nitjsr.ac.in )

National Institute of Technology Jamshedpur

Soufiane Bentout

University of Algereia

Mohammed Tarik Touaoula

Hassiba Benbouali University of Chlef: Universite Hassiba Benbouali de Chlef

\section{Research Article}

Keywords: COVID-19, Age-structured, SIR model, Basic reproduction number, Peak epidemic

Posted Date: June 29th, 2021

DOl: https://doi.org/10.21203/rs.3.rs-253566/v1

License: (c) (i) This work is licensed under a Creative Commons Attribution 4.0 International License.

Read Full License 


\title{
Analyzing the spread of COVID-19 disease using an age-structured model: Application to Italy, Spain, France, UK, and Algeria using early data
}

\author{
Salih Djilali · Sunil Kumar · Soufiane Bentout · Mohammed Tarik Touaoula.
}

Received: date / Accepted: date

\begin{abstract}
The outbreak epidemic of the coronavirus (COVID19) puts the whole world in an alert stage due to the highly spread speed. Recent researches prove that the immunity system of the human being has a crucial role in recovering. It is known that the immunity system becomes weaker for older persons. Hence, the coronavirus is highly risked for aged individuals mostly the ones that passed the 60s. The most recent approximations neglect the role of age of the individuals in the spread and degree of the fatality of the COVID-19 virus. The recent statistics show a very high death number due to COVID-19 for aged individuals. Here, we propose an age-structured model for analyzing the peak outbreak epidemic and give an approximative time of this peak next to the number of death cases due to the COVID19 in Italy, Spain, France, United Kingdom (UK), and Algeria using early data. Further, we show the effect of the governmental restrictions of social movements on this peak and also we provide an approximative time of the end of this infection with (resp. without) restriction.
\end{abstract}

Keywords COVID-19 · Age-structured · SIR model · Basic reproduction number $\cdot$ Peak epidemic

\section{S.Djilali}

Laboratoire d'Analyse Non Linre et Mathtiques Appliqu. , Universitou Bakr Belka Tlemcen 13000, Algeria.

Faculty of Exact sciences and informatics, Mathematic Department, Hassiba benbouali university, Chlef, Algeria.

E-mail: djilali.salih@yahoo.fr, s.djilali@univ-chlef.dz

S.Kumar

Department of Mathematics, National Institute of Technology, Jamshedpur 831014, Jharkhand, India

E-mail:skumar.math@nitjsr.ac.in

S.Bentout

Laboratoire d'Analyse Non Linre et Mathtiques Appliqu. , Universitou Bakr Belka Tlemcen 13000, Algeria.

Department of Mathematics and Informatics,Center of Belhadj Bouchaib Ain Temouchent,BP 284 RP, 46000, Algeria.

E-mail:bentouts of iane@gmail.com

M.T.Touaoula

Laboratoire d'Analyse Non Linre et Mathtiques Appliqu. , Universitou Bakr Belka Tlemcen 13000, Algeria.

Drtement de Mathtiques, Universitou Bakr Belka Tlemcen 13000, Algeria.

E-mail:t_touaoula@yahoo.fr

\section{Highlights}

- A COVID-19 age-structured model is considered.

- Approximating the model parameters based upon the available data of WHO.

- Some results of the peak epidemic outbreak are given.

- Approximative time of the end of the epidemic is estimated.

- The number of death cases is approximated.

- The effect of the full restriction of social movements on the spread and the peak of coronavirus epidemic.

\section{Introduction}

Recently, at the end of 2019, a new disease has hit the world, known as COVID-19. It started in Wuhan city in China. This infection is known by its highly spread speed, wherein a very small time it outbreaks into every corner of our planet. The new COVID-19 is considered a respiratory virus which spreads primarily through droplets generated when infected individual coughs or sneezes, or through droplets of saliva or discharge from the nose. These droplets can be found on surfaces in public places as metros, buses, and other places. It is the reason for putting strict measures of restriction of different social movements. Until now there is no known completely safe treatment or vaccines whereas COVID-19 is spreading very fast in every corner of the world, Hence, The World Health Organization declares it as a pandemic. All the planet struggle to reduce the outbreak of this infectious disease such as Italy, France, UK, USA, Iran, Algeria, Spain, and others. There is a huge financial deficit due to this disease which shows the huge impact on the economical system of the world. Many countries declare the third stage of restriction, the highest rate of mortality is in Italy with $9.2 \%$ of death after infection. Very recently, researches in [11] prove that the individuals that mostly die due to COVID-19 are the older people, where this research shows that the people in the 60 s have a $0.4 \%$ probability of dying, whereas individuals in 70 s have a $1.3 \%$ probability of death. However, individuals age over 80 has a $3.6 \%$ chance of dying. It seems that this is not a high rate of death but in Italy, during the recent outbreak $83 \%$ of the death are aged over the 60s [12]. Many 
new models study the outbreak of the coronavirus as the papers [9,8,5,6], but they neglect this case of death, and focus on the number of the infected individuals only. To mention that the declared cases are mostly the old individuals, where the researches show that a human can have COVID-19 with non-well visual symptoms. Thus, our objective is to determine the number of infected individuals and the death in Italy, Spain, France, UK, Algeria sing early data and showing the number of infection cases that could be obtained without the measures taken by governments. Also, we will give an approximative time for the end of this coronavirus epidemic in the previously mentioned countries and the peak epidemic outbreak with the help of these early data and the age-structured model. This result is very helpful for avoiding the second wave tat the world wideness in these days and helps in giving proper measures to limit the spread of this highly infectious disease, which obligate the decisionmakers to apply some other measures for avoiding the big increase of infection cases. Indeed, most part of countries in different corners of the planet obligates their citizens to full restriction of the social groupings to limit even to vanish the transmission which is known by stage 3 . Our second purpose is to analyze the effect of stage 3 on the spread of COVID19. Furthermore, we will also mathematically compute the proportion of the susceptible individuals after the end of this pandemic. Also, the considered necessary data are given in Fig. 1, 3 and the tables 1, 2, 3 these data are obtained through the references [14, 15, 16, 17, 18, 19].

\section{Model and estimation of parameters}

As been presented in the introduction section, the age of the individuals plays an important role in the mortality done by COVID-19. The age-structured models have been used in the past years to understand the spread of infectious diseases we refer as example the papers [1,3, 2, 10]. Hence, motivated by the statistics and the biological background of aged structured models we propose the following age-structured model:

$$
\left\{\begin{array}{l}
\frac{\partial S(t, a)}{\partial t}+\frac{\partial S(t, a)}{\partial a}=-\beta S(t, a) \int_{0}^{+\infty} I(t, \theta) d \theta \\
\frac{\partial I(t, a)}{\partial t}+\frac{\partial I(t, a)}{\partial a}=\beta S(t, a) \int_{0}^{+\infty} I(t, \theta) d \theta-(\gamma+\delta(a)) I(t, a), \\
\frac{d D(t)}{d t}=\int_{0}^{+\infty} \delta(a) I(t, a) d a \\
\frac{d R(t)}{d t}=\gamma \int_{0}^{+\infty} I(t, a) d a \\
S(t, 0)=0, \quad I(t, 0)=0, \\
S(0, a)=S_{0}(a) \in L^{1}(0,+\infty), \quad I(0, a)=I_{0}(a) \in L^{1}(0,+\infty),
\end{array}\right.
$$

where $S(t, a), I(t, a)$ stands for the fraction of the susceptible density and the infected density, this means that $0 \leq S(t, a), I(t, a) \leq 1 . D(t)$ is the reported death cases due to the coronavirus at time $t . R(t)$ is the removed individuals (or recovered) at time $t$. $a$ represents the age of the individuals which is measured in years. The time $t$ is measured in days. $\beta$ is the transmission rate. $\gamma$ represents the removal rate. $1 / \gamma$ is the average of the infection period which is estimated for COVID-19 as 10 to 14 days. In [13,6] it is considered that $\gamma=0.1$, which means that the average of the infection period is 10 days. $\int_{0}^{+\infty} I(t, a) d a$ stands for the total fraction of the infected individuals at time $t$. To mention that the boundary conditions $S(t, 0)=0, \quad I(t, 0)=0$ represents that there are no newborns (or no immigrations), these assumptions are put due to stopping all kind of immigrations for the most part of countries in our planet and neglecting the number of the new borne. Based on the statistics mentioned in the introduction section, we can consider that the mortality rate of the infected individuals with the age over $60 \mathrm{~s}$ is $\delta^{*}=5.3 \%$, for the individual with age under 60 s has $0.01 \%$ chance of dying if infected, which means that the mortality of the infected individuals under the age of 60s due to coronavirus can be neglected. Hence the functional $\delta(a)$ can be expressed in the following manner:

$\delta(a)=\left\{\begin{array}{l}\delta^{*} \quad \text { if } \quad a \geq \tau, \\ 0 \quad \text { if } \quad a<\tau,\end{array}\right.$

with $\tau=60$. For the choice of the initial data, we will be interested in determining the fraction of the susceptible and the infected individuals who are under 60 years old (resp. over 60), for the purpose of determining the fatality degree of the COVID-19 in each one of the studied samples. Also, we are interested to determine the proportion of the aged individuals that have passed the 60s in the susceptible and infected categories. In Table 3 we offer the percentage of the aged individuals (over 60s) in Italy, Spain, France, UK, and Algeria. Based on Table 3 we split the infected individuals and the susceptible individuals at $t=0$ using Table 3 as for example in Italy we put $36.68 \% \times S_{0}(a)$ are aged individuals and $63.32 \% \times S_{0}(a)$ are young individuals, and in the next section we will see the effect of this split on the degree of the fatality of COVID-19 in each studied countries.

We define the following space

$$
\mathfrak{H}=L^{1}(0,+\infty) \times L^{1}(0,+\infty) \times \mathbb{R}^{+} \times \mathbb{R}^{+},
$$

with $L^{1}(0,+\infty)$ is the space of functions that are non-negative and Lebesgue integrable, equipped with the following norm

$\|(\phi, \psi, x, y)\|_{\mathfrak{H}}=\int_{0}^{+\infty}|\phi(\theta)| d \theta+\int_{0}^{+\infty}|\psi(\theta)| d \theta+|x|+|y|$,

We assume that the initial conditions $\left(S_{0}, I_{0}, D_{0}, R_{0}\right) \in \mathfrak{H}$. By using [7,1], it can be obtained that system 2.1] has a unique positive solution for initial conditions $\left(S_{0}, I_{0}, D_{0}, R_{0}\right) \in \mathfrak{H}$

For understanding the spread of the COVID-19 we must calculate the basic reproduction number $R_{0}$. At first, we define the probability $P(a)$ of an infected individual with age $a$ to still remains in the infected class. Hence, this probability is expressed as:

$$
\left\{\begin{array}{l}
P^{\prime}(a)=-\gamma P(a)-\delta(a) P(a), \\
P(0)=1
\end{array}\right.
$$

Then, $P(a)=\mathrm{e}^{-\gamma a-\int_{0}^{a} \delta(a) d a}$. We know that $R_{0}=c p \Delta t$; where $c$ is the achieved contact between the $S$ class and the infected one, $p$ the probability of transmission and $\Delta t$ is the period of infection. For the model 2.1] we have $c p=\beta$, and $\Delta t=\int_{0}^{+\infty} \mathrm{e}^{-\gamma a-\int_{0}^{a} \delta(\theta) d \theta} d a$. Therefore, The basic reproduction number is expressed as:

$R_{0}=\beta \int_{0}^{+\infty} \mathrm{e}^{-\gamma a-\int_{0}^{a} \delta(\theta) d \theta} d a$ 

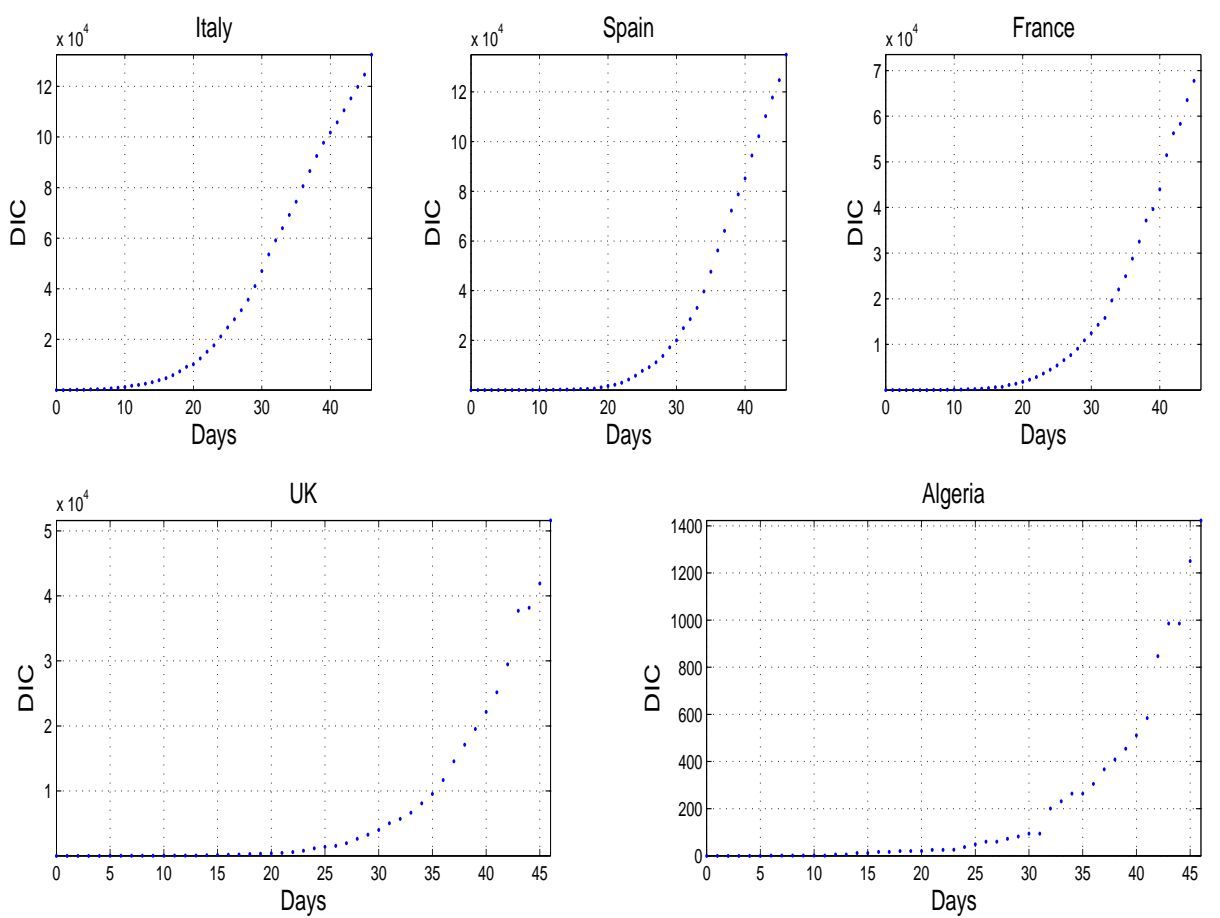

Fig. 1: The evolution of the number of the declared infected cases (DDC) in Italy, Spain, France, UK and Algeria between $t=0(20 / 02 / 2020)$ and $t=46(06 / 04 / 2020)$

Simplifying the previous formula of the basic reproduction number in the following manner

$$
\begin{aligned}
R_{0} & =\beta\left(\int_{0}^{\tau} \mathrm{e}^{-\gamma a} d a+\int_{\tau}^{+\infty} \mathrm{e}^{-\gamma a-\int_{0}^{a} \delta(\theta) d \theta} d a\right), \\
& =\beta\left(\frac{1}{\gamma}\left(1-\mathrm{e}^{-\gamma \tau}\right)+\int_{\tau}^{+\infty} \mathrm{e}^{-\gamma a-\delta^{*}(a-\tau)} d a\right),
\end{aligned}
$$

By a straightforward calculation we get,

$R_{0}=\frac{\beta\left(\delta^{*}+\gamma-\delta^{*} \mathrm{e}^{-\gamma \tau}\right)}{\gamma\left(\gamma+\delta^{*}\right)}$

Now, it remains to determine the value of the transmission rate $\beta$. In fact, in [8,9] it is shown that there is a useful method for determining this transmission rate for ordinary differential equations. Here we will use a similar method for the partial differential equations. At first, the total densities of the population in Italy, Spain, France, UK, and Algeria mentioned in Table 1 the data mentioned in Fig. 1 are normalized and used in Fig. 3. Ones we integrate the second equation of the model 2.1 we get:

$\frac{d i(t)}{d t}=(\beta s(t)-\gamma) i(t)-\int_{\tau}^{+\infty} \delta(a) I(t, a) d a$,

with $i(t)=\int_{0}^{+\infty} I(t, a) d a, s(t)=\int_{0}^{+\infty} s(t, a) d a$. we expect that $i(t)$ (the total proportion of infected individuals) has the following special form:

$i(t)=x_{1} \mathrm{e}^{x_{2} t}$

Using the values of $x_{1}, x_{2}$ obtained in Table 2 we can determine the value of the transmission rate $\beta$, which by taking a look at 2.4) it is difficult to deduce it. Thus, using the fact that $0 \leq \delta(0) \leq \delta^{*}$ we can obtain that:

$$
\beta_{1} \leq \beta \leq \beta_{2}
$$

where $\beta_{1}=\frac{x_{2}+\gamma}{s(0)}, \beta_{2}=\frac{x_{2}+\gamma+\delta^{*}}{s(0)}$. On the other hand the choice of the transmission rate $\beta$ is highlighted in Table 4

\section{Approximating the final size of the susceptible population}

Integrating the S-class and the I-class with respect to the age in 2.1. we get the following system

$\left\{\begin{array}{l}s^{\prime}(t)=-\beta s(t) i(t), \\ i^{\prime}(t)=\beta s(t) i(t)-\gamma i(t)-\int_{0}^{+\infty} \delta(a) I(t, a) d a, \\ \frac{d D(t)}{d t}=\int_{0}^{+\infty} \delta(a) I(t, a) d a, \\ \frac{d R(t)}{d t}=\gamma i(t),\end{array}\right.$

with $s(t)=\int_{0}^{\infty} S(t, a) d a$ and $i(t)=\int_{0}^{\infty} I(t, a) d a$. Add the first equation and the second equation of (3.1) to obtain

$s(t)+i(t)+\gamma \int_{0}^{t} i(\sigma) d \sigma+\int_{0}^{t} \int_{0}^{\infty} \delta(a) I(\sigma, a) d a d \sigma=s_{0}+i_{0}$,

which implies $i(t)<\infty$ for $t \geq 0$ and $\int_{0}^{\infty} i(t) d t<\infty$. From the first equation of 3.1 we have

$\ln \frac{s(t)}{s_{0}}=-\beta \int_{0}^{t} i(\sigma) d \sigma$, 

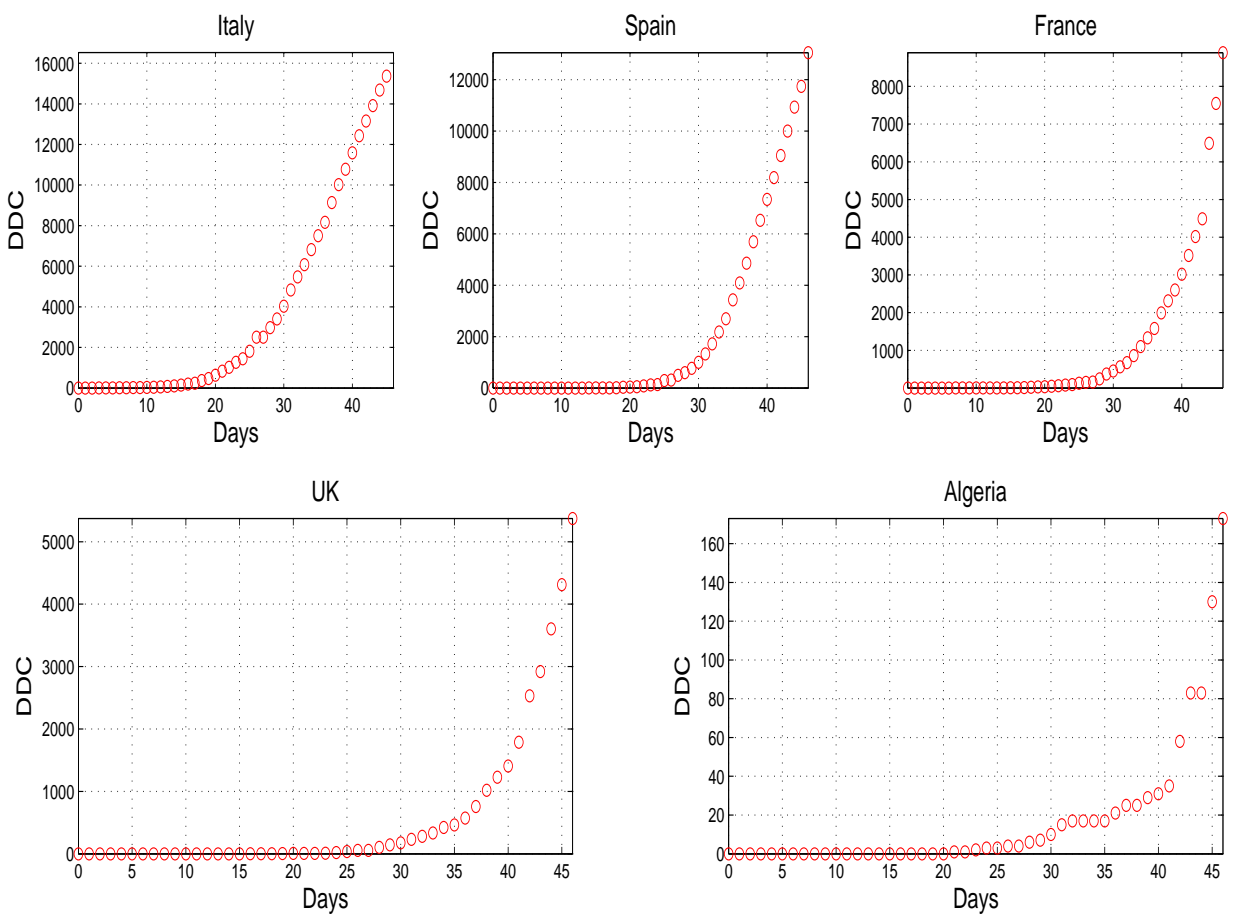

Fig. 2: The evolution of the number of the declared death cases (DDC) due to COVID-19 in Italy, Spain, France, UK and Algeria between $t=0(20 / 02 / 2020)$ and $t=46(06 / 04 / 2020)$

\begin{tabular}{llllll}
\hline \hline Country & Italy & Spain & France & U.K. & Algeria \\
\hline \hline Total number of population (million) & 60.48 & 44.66 & 66.49 & 66.44 & 41.32 \\
\hline \hline
\end{tabular}

Table 1: Population densities in the studied samples.

\begin{tabular}{lll}
\hline \hline Country & $x_{1}$ & $x_{2}$ \\
\hline \hline Italy & $3.4982 \times 10^{-4}$ & 0.09 \\
Spain & $1.2882 \times 10^{-4}$ & 0.15 \\
France & $6.6834 \times 10^{-5}$ & 0.13 \\
UK & $1.7219 \times 10^{-5}$ & 0.18 \\
Algeria & $8.9545 \times 10^{-7}$ & 0.285 \\
\hline \hline
\end{tabular}

Table 2: Table Estimation of the parameters using the available data in Fig. 1

\begin{tabular}{llllll}
\hline \hline Country & Italy & Spain & France & U.K. & Algeria \\
\hline \hline over 55s & $36.08 \%$ & $31.48 \%$ & $32.93 \%$ & $31.21 \%$ & $13.58 \%$ \\
\hline \hline
\end{tabular}

Table 3: The percentage of the aged individuals in Italy, France, Spain, UK, Algeria see [20]

\begin{tabular}{llllll}
\hline \hline Country & Italy & Spain & France & U.K. & Algeria \\
\hline \hline$\beta$ & 0.2407 & 0.2963 & 0.2815 & 0.3315 & 0.394 \\
\hline \hline$\beta_{1}$ & 0.1909 & 0.2465 & 0.2313 & 0.2314 & 0.389 \\
\hline \hline$\beta_{2}$ & 0.2412 & 0.2968 & 0.2816 & 0.332 & 0.4395 \\
\hline \hline
\end{tabular}

Table 4: The choice of the transmission rate in Italy, France, Spain, UK, Algeria used in sect 4

and

$s(t)=\exp \left(-\beta \int_{0}^{t} i(\sigma) d \sigma\right)$
Since $s(t)$ is decreasing and $\int_{0}^{\infty} i(t) d t<\infty, \lim _{t \rightarrow \infty} s(t)=$ $s(\infty)>0$. Moreover, according to 3.2 the limit of $i(t)$ as $t$ goes to infinity exists and by $\int_{0}^{\infty} i(t) d t<\infty, \lim _{t \rightarrow+\infty} i(t):=$ $i(\infty)=0$. Passing to the limit in 3.2 ,

$\ln \frac{s_{0}}{s(\infty)}=\beta \int_{0}^{+\infty} i(t) d t$

From the third and fourth equations of 3.1 combining with the (3.2) we deduce that $R$ and $D$ have also a finite limit denoted $r(\infty)$ and $d(\infty)$, respectively. Note that $r(\infty)$ and $d(\infty)$ represent the total fraction of the recovered individuals and the fraction of the death cases after the end of the pandemic, respectively. Set $s_{0}=\int_{0}^{+\infty} S_{0}(a) d a, i_{0}=\int_{0}^{+\infty} I_{0}(a) d a, r_{0}=$ $R(0), d_{0}=D(0)$. Integrating the third equation of (3.1) and passing to the limit, from 2.2 we obtain

$$
\begin{aligned}
d(\infty)-d_{0} & =\int_{0}^{+\infty} \int_{0}^{+\infty} \delta(a) I(t, a) d a d t \\
& =\delta^{*} \int_{0}^{+\infty} \int_{\tau}^{+\infty} I(t, a) d a d t \\
& =\delta^{*}\left(\int_{0}^{+\infty} i(t) d t-\int_{0}^{+\infty} \int_{0}^{\tau} I(t, a) d a d t,\right)
\end{aligned}
$$

In view of 3.4 we find

$$
d(\infty)-d_{0}=\frac{\delta^{*}}{\beta} \ln \frac{s_{0}}{s(\infty)}-\delta^{*} \int_{0}^{+\infty} \int_{0}^{\tau} I(t, a) d a d t
$$



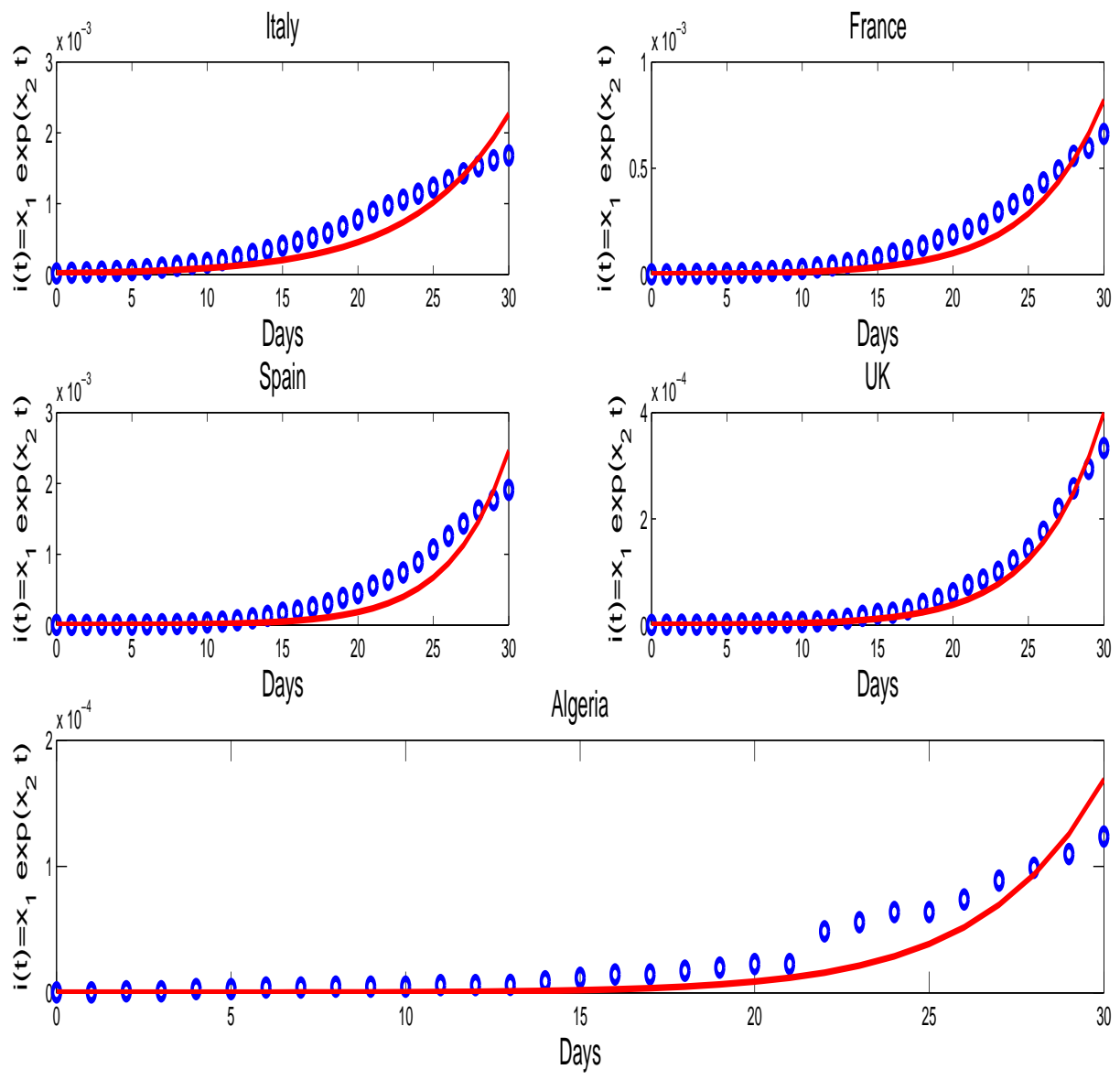

Fig. 3: The method of approximating $x_{1}$ and $x_{2}$ using the data in Fig. 1 where the values used in Table 2 are used for each sample where $t=0$ represents the date $15 / 03 / 2020$.

\begin{tabular}{llllll}
\hline \hline Country & Italy & Spain & France & U.K. & Algeria \\
\hline \hline$R_{0}$ & 2.405 & 2.9606 & 2.8127 & 3.3123 & 3.9367 \\
\hline \hline
\end{tabular}

Table 5: The basic reproduction number in Italy, France, Spain, UK, Algeria

Similarly, for the fourth equation of 3.1 and 3.4 we get

$$
\begin{aligned}
r(\infty)-r_{0} & =\gamma \int_{0}^{+\infty} i(t) d t, \\
& =\frac{\gamma}{\beta} \ln \frac{s_{0}}{s(\infty)} .
\end{aligned}
$$

Further, 3.2, 3.4, 3.5, 3.6 imply

$s(\infty)+\left(\frac{\gamma+\delta^{*}}{\beta}\right) \ln \frac{s_{0}}{s(\infty)} \leq s_{0}+i_{0}$.

Finally using 2.3 we arrive at

$s(\infty)+\left(\frac{\delta^{*}\left(1-e^{-\gamma \tau}\right)+\gamma}{\gamma R_{0}}\right) \ln \frac{s_{0}}{s(\infty)} \leq s_{0}+i_{0}$.

\section{Understanding the spread of COVID-19 disease in Italy, France, Spain, Uk and Algeria using model 2.1)}

In this section, we will investigate the number of infection cases of COVID-19 disease and provide the worst-case scenario that could be happened in the mid year 2020. In fact, we will split our section into the following section for discussing each country separably:

\subsection{The spread of COVID-19 in Italy}

Using the numerical simulation and the previously mentioned values of the parameters we obtain the obtained results highlighted in Fig. 4. Indeed, we can mention the following scenario that could happen in the case of the absence of the measures taken by the Italian government in the last few months

For the upper left figure of Fig. 4 we can see the good fitting of data and the approximative infection cases (black circles for the exact number of infection cases) and the total fraction of the infected individuals are highlighted in the blue curve. The peak of the infection reached for Italy at $t=41$ (at 25/04/2020). In this date, the proportion of the infected individuals is 0.04238 (which means that 2563100 active infected individuals) which is a huge proportion. On the 
other hand, based on Fig. 4 we deduce that COVID-19 disease could end at $t=90$ (which means by the end of June).

In fact, we are interested in the mortality due to COVID19 in Italy, using the upper right-hand figure in Fig. 4 we can see the good agreement with the data (which are highlighted in green circles). Further, by the end of June our numerical simulation (Fig. 4) notice that the infected proportion of the death due to COVID-19 is 0.005995 (which means 362580 death cases). Furthermore, using the lower figure of Fig. 4 we show that the infection will reach mostly older individuals. This result agrees with what we have in the reality. Indeed, the symptoms appear in older individuals more than younger ones where the immunity system of the younger ones is more effective than the older ones.

\subsection{The spread of COVID-19 in Spain}

Using the numerical simulations and the previously mentioned values of the parameters we obtain the results highlighted in Fig. 5. Indeed, we can present the following scenario

The same remark as for Italy we can see through Fig. 5 the good fitting of the solution of the model 2.1 with data, the real data (in a black circle for the infection cases and the green circles for the death cases) lies over the predictive proportion of the infection cases (resp. the proportion of the death cases). The peak of the COVID-19 epidemic will be reached in Spain at $t=50$ (at $05 / 05 / 2020$ ). On this date, the proportion of the infection cases is 0.1643 (which means that 7337600 active infected individuals) which is a huge proportion. On the other hand, based on Fig. 4 we deduce that COVID-19 disease will end at $t=90$ (which means by the end of June).

Indeed, we are involved in studying the mortality done by COVID-19 in Spain. By the beginning of June, we conclude that the infected proportion of death by COVID-19 is 0.02946 (which means 100000 death cases). The same remark obtained in the lower figure of Fig. 4 can be expanded for the lower figure of Fig. 5 .

\subsection{The spread of COVID-19 in France}

Using the numerical simulation and the previously mentioned values of the parameters we obtain Fig. 6. Also, we get the following results:

Using Fig. 6 we can say the spread peak epidemic of the COVID-19 in France going to be very high, This peak will be reached at $\mathrm{t}=50$ (which means at $05 / 05 / 2020$ ) with 3037900 infection cases. We also deduce that the infection will be finished after 92 days starting from 15/03/2020, which means by the beginning end of June. Note that our numerical simulations highlight that this infection will take 433510 death if the situation stays the same.

\subsection{The spread of COVID-19 in UK}

Using the numerical simulations and the previously mentioned values of the parameters we obtain Fig. 7 The obtained results are:

Using Fig. 7 we can mention that the spread Peak epidemic of the COVID-19 in the UK, which fits with data, and with a very good precision see the black and the green circles in Fig. 7. In fact, the peak epidemic outbreak will be reached at $t=54$ (which means at 25/04/2020), the extinction of the COVID-19 from the population of UK will be after 94 days after $05 / 05 / 2020$ which means by the beginning of Jun. Indeed, on the date 25/04/2020 (at the peak date) we can deduce that the number of infected individuals will be very high (by 683670 individuals).

\subsection{The spread of COVID-19 in Algeria}

Using the numerical simulation and the previously mentioned values of the parameters we obtain Fig. 8 . The obtained results are:

Using Fig. 8 we can determine the spread Peak epidemic of the COVID-19 in Algeria, which fits with the data, and with a very good precision see the black and the green circles in Fig. 8. In fact, the peak epidemic outbreak could be reached at day $t=58$ (which means at 12/05/2020), the extinction of the COVID-19 from the population of Algeria done after 96 days after 15/03/2020 which means by the end of June. Indeed, in the date $05 / 05 / 2020$ (at the peak date) we can highlight that the number of infected individuals will be dangerously high (by 2854400 individuals).

\section{The effect of the full restriction on the peak and period of COVID-19 outbreak epidemic}

In this section, we are interested in analyzing the effect of the full restriction of the social movement obligated by the governments. In this case, it is wise to consider that the transmission rate is a function of time, where after this restriction, the transmission will be highly decreased. In [9], the following transmission rate is investigated:

$\beta(t)=\left\{\begin{array}{l}\beta_{0} \text { if } t<T \\ \beta_{0} \mathrm{e}^{-\mu(t-N)} \text { if } T<t,\end{array}\right.$

In fact we consider that $\beta_{0}$ takes the values highlighted in Table 4 next to $\mu=0.16$ (see [9]) and $T=37$ (this restriction is assumed to be taken at 19/04/2020). The effect of this transmission rate on the Peak outbreak epidemic of COVID19 is highlighted in Fig. 9

Indeed, the black curves are the proportion of infection cases shown in Fig. 4, 5, 6, 7, 8, The blue curves are the proportion of the infection cases after the full restriction of social movements using the transmission functional (5.1). These restriction measures are assumed to be started at the $t=37$ from 15/03/2020 (which means these measures are taken on 19/04/2020). It is obvious to see that this measure reduces 


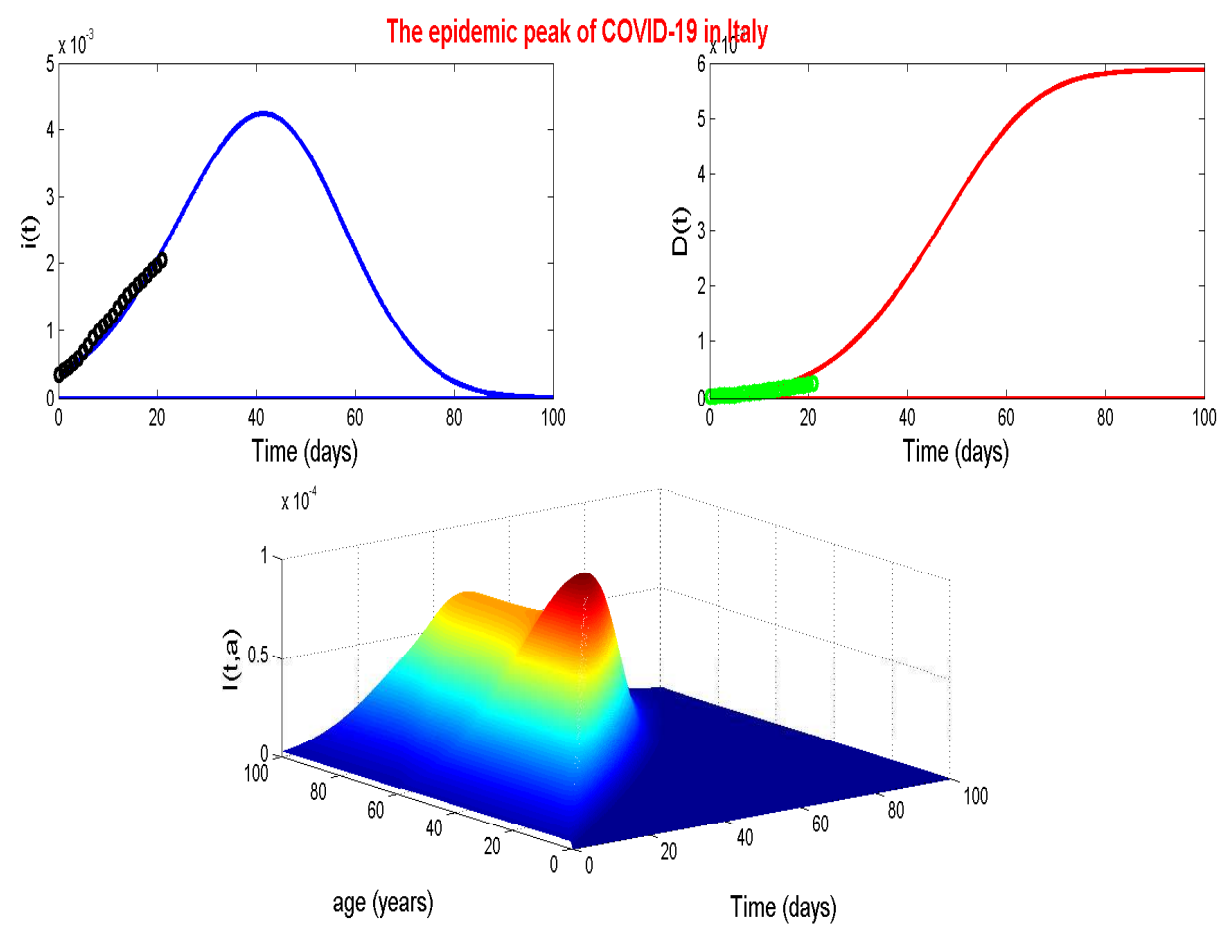

Fig. 4: Numerical simulation of the spread of COVID-19 epidemic in Italy
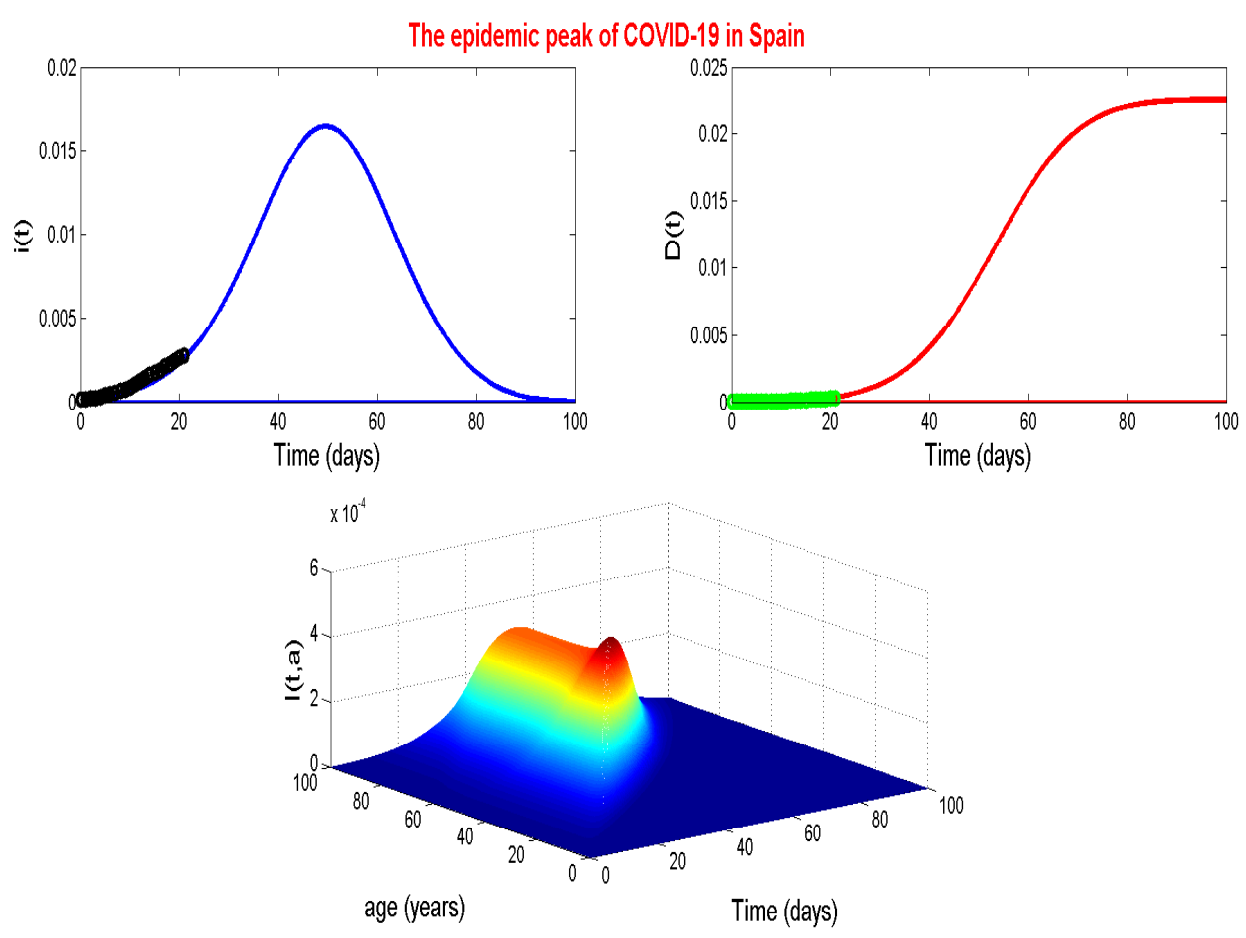

Fig. 5: Numerical simulation of the spread of COVID-19 epidemic in Spain

the peak of the epidemic outbreak and the period, more results are provided in Table 6 As a first remark, we can see that after the full restrictions taken at $t=45$ the infection will disappear at day 69-70, which means that 20-30 days of full restriction is able to stop the spread of infection

\section{Conclusion}

In this work, we investigate some results of the coronavirus outbreak epidemic using an age-structured model. The reason behind introducing the age effect in our system 2.1 is 

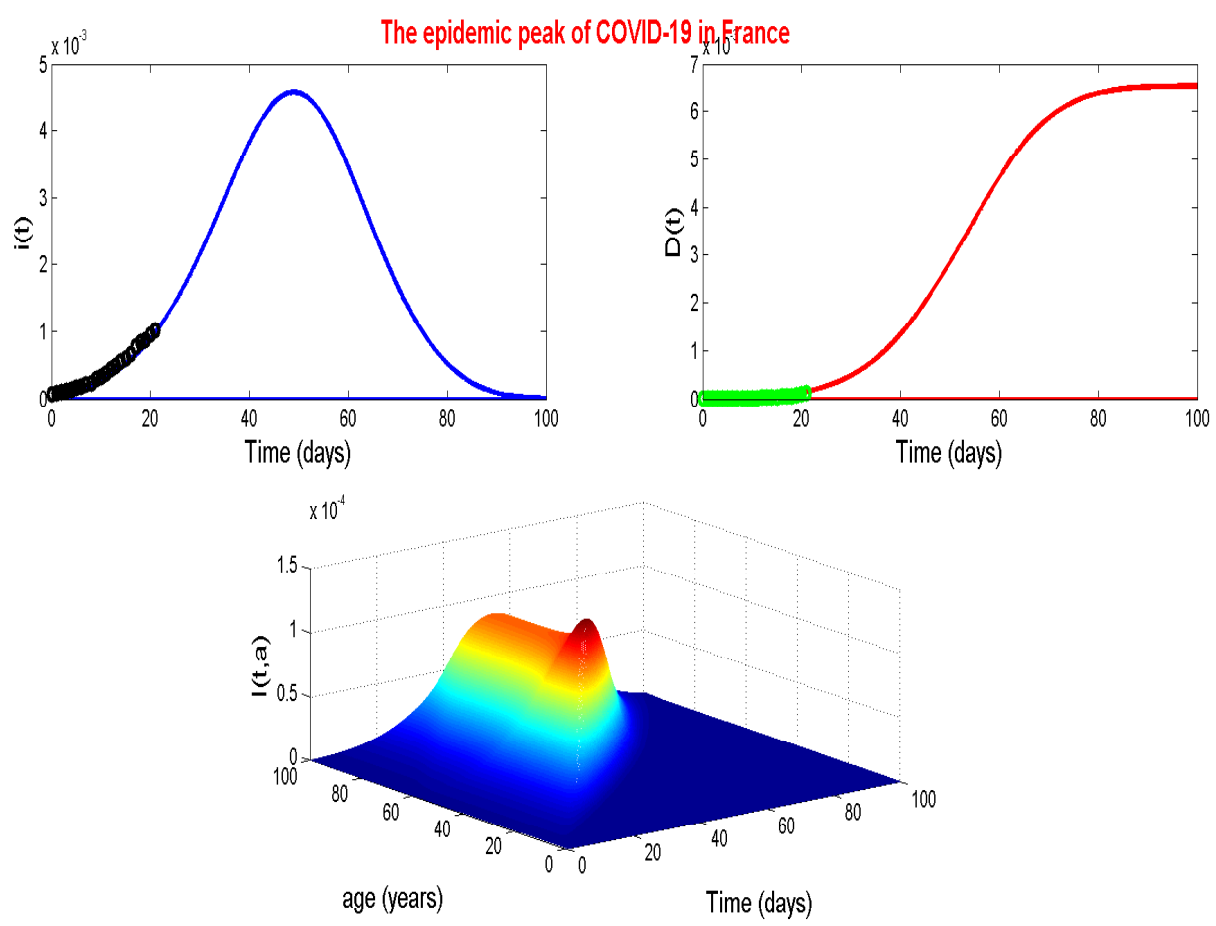

Fig. 6: Numerical simulation of the spread of COVID-19 epidemic in France

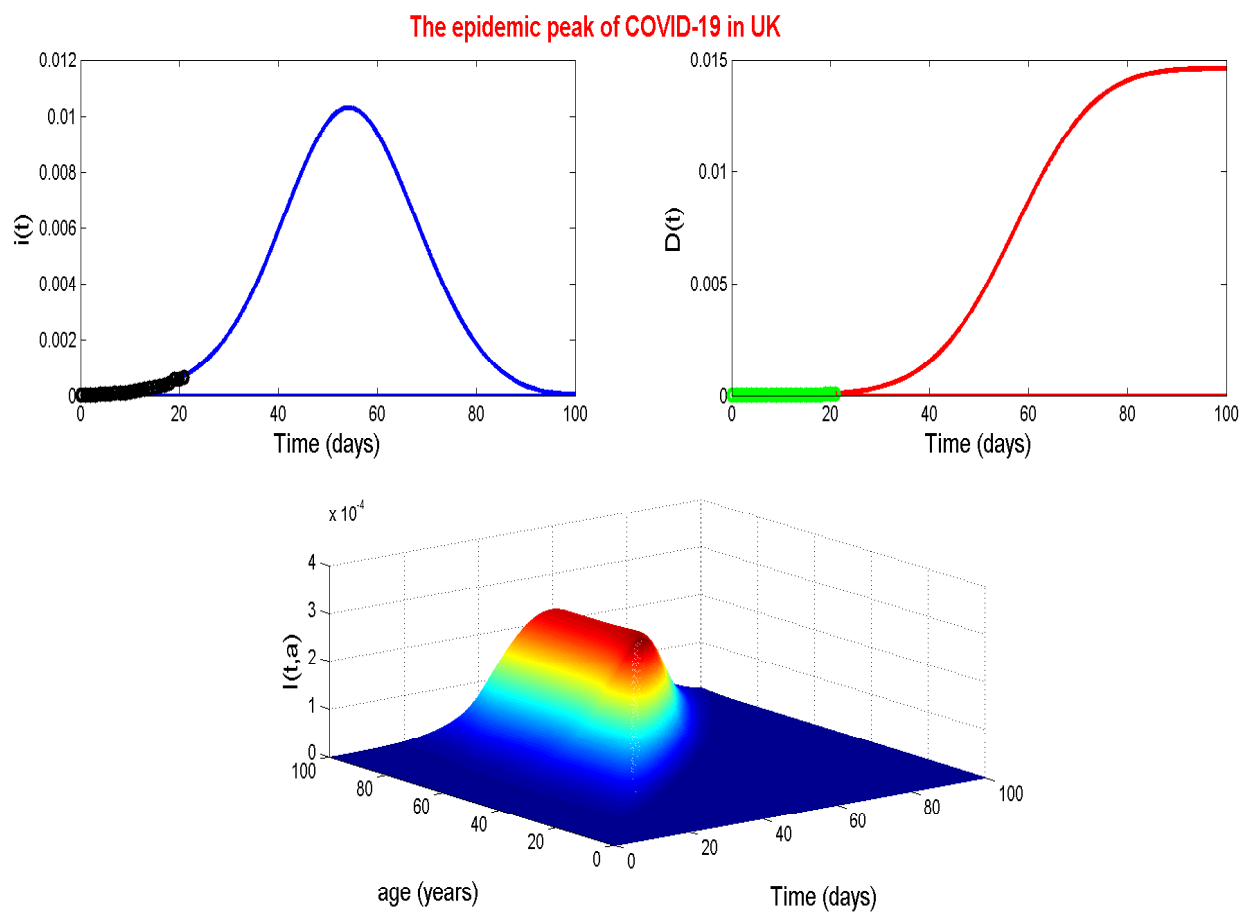

Fig. 7: Numerical simulation of the spread of COVID-19 epidemic in UK

to model the witnessed big mortality due to the COVID-19. In fact, the most part of this mortality is for the aged individuals that have passed the 60s. The primary interest of this work is to determine the highest number of infection cases Italy: This country is the one which has the worst scenario, the mortality cases are very high. In fact, in [9] It is obtained that can the world witness and the approximative time of this peak. This study is applied to five countries 


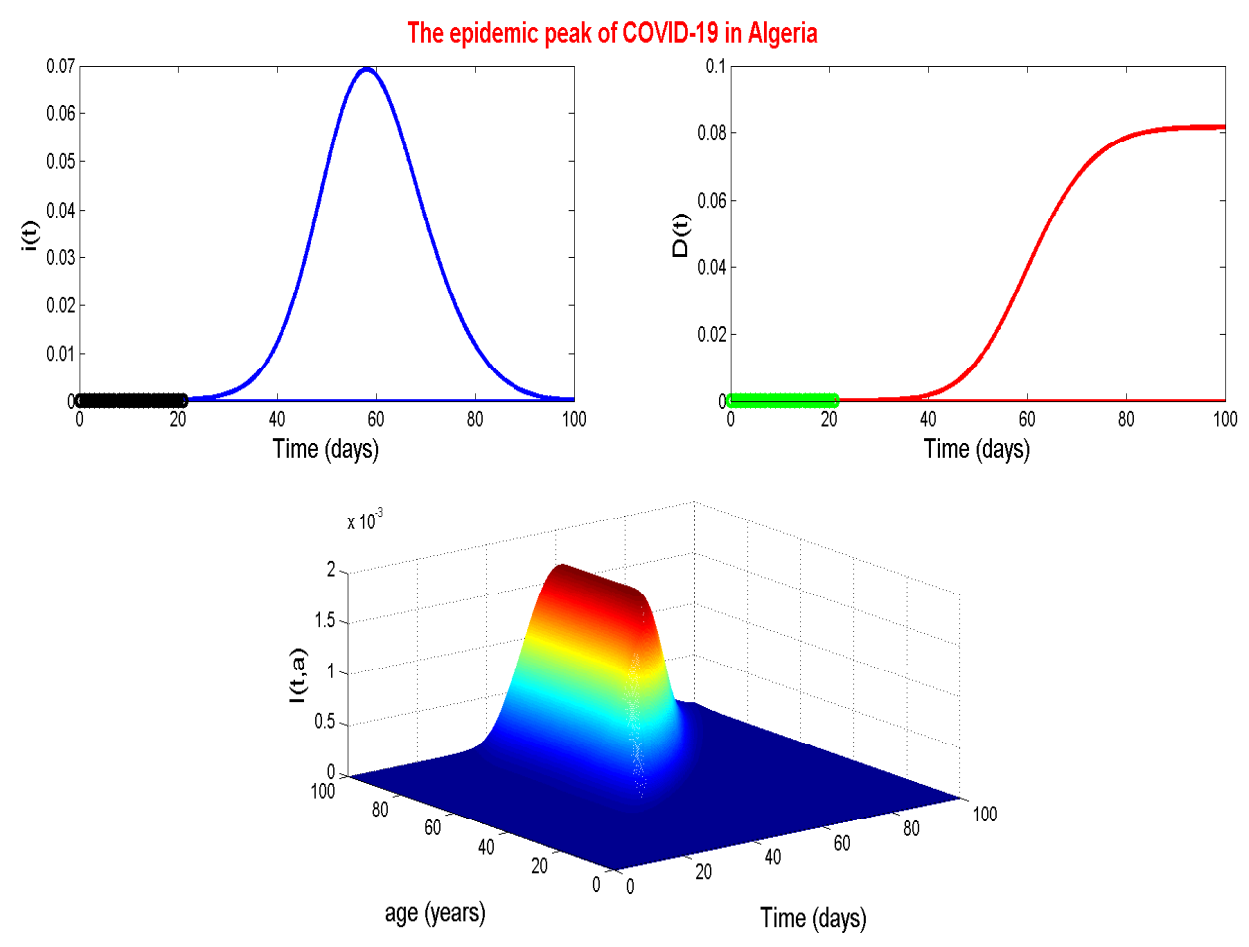

Fig. 8: Numerical simulation of the spread of COVID-19 epidemic in UK
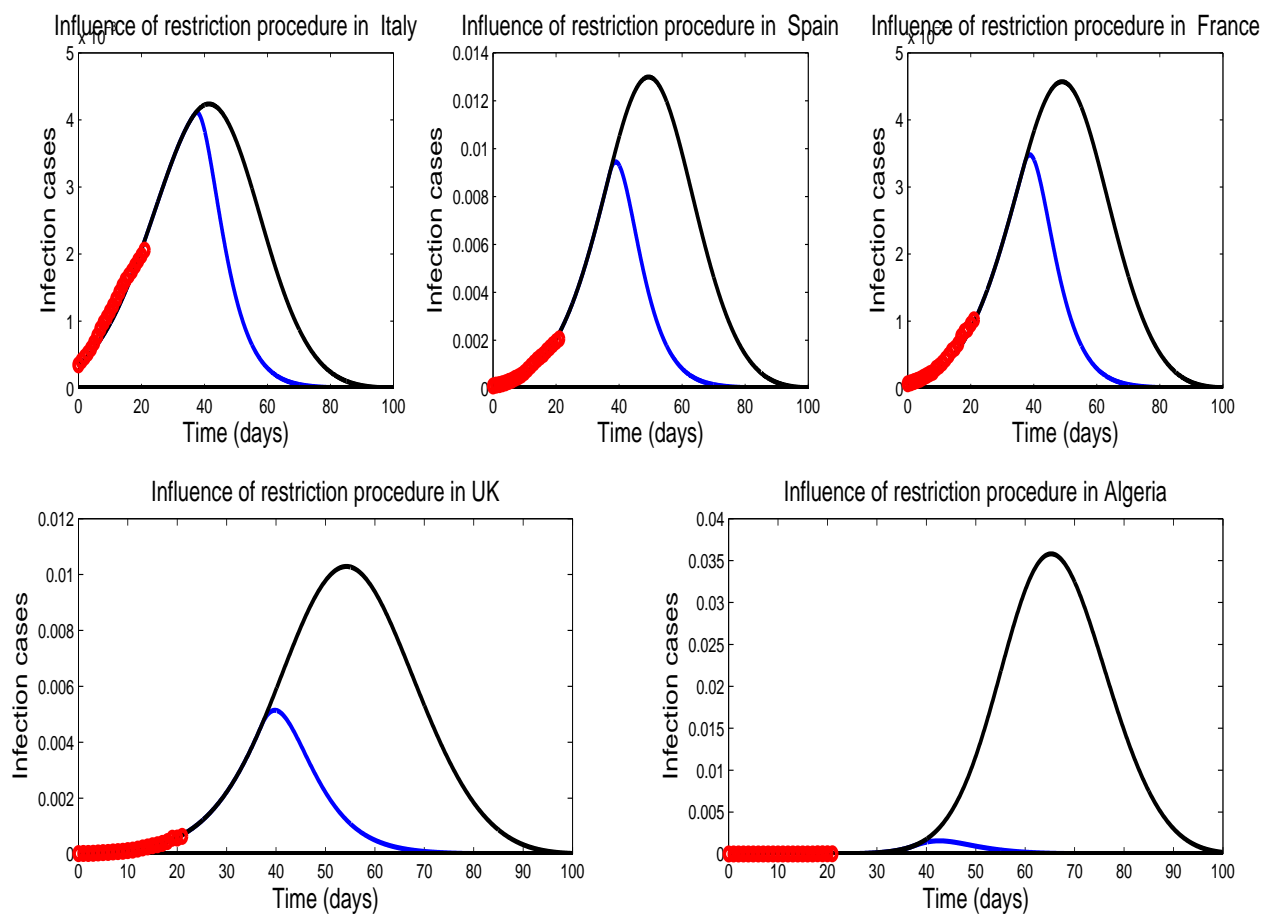

Fig. 9: Numerical simulation of the spread of COVID-19 epidemic in Italy, Spain, France, UK and Algeria after a full restriction of social movement

that $R_{0}=3.79$ (with data before $14 / 03 / 2020$ ), however, in the updated data (for data before 06/04/2020) the basic reproduction is decreased to $R_{0}=2.405$ which means that there was a decrease in the speed of COVID-19 spread.
Also, based on our results concerning the peak epidemic it is been reached on 25/04/2020 with 2563100 infection cases. The epidemic will be finished by the end of May with 362580 death cases and we can mention that most 


\begin{tabular}{llllll}
\hline \hline Country & Italy & Spain & France & UK & Algeria \\
\hline \hline PEB without restriction (NII) & $41(256310)$ & $50(733376)$ & $50(433510)$ & $54(683670)$ & $58(2854400)$ \\
\hline \hline PEB with restriction (NII) & $37(249240)$ & $37(425300)$ & $37(234040)$ & $37(341100)$ & $37(65120)$ \\
\hline \hline PTEE without restriction (NII) & 90 & 90 & 92 & 94 & 98 \\
\hline \hline PTEE with restriction (NII) & 70 & 70 & 70 & 69 & 55 \\
\hline \hline
\end{tabular}

Table 6: Some conclusions about the Peak Epidemic Outbreak (PEB) and the approximative time of the End of the Epidemic (PTEE) with (resp. without) restriction next to the Number of the Infected Individuals (NII) at each peak epidemic outbreak , note that $t=0$ refer to $15 / 03 / 2020$.
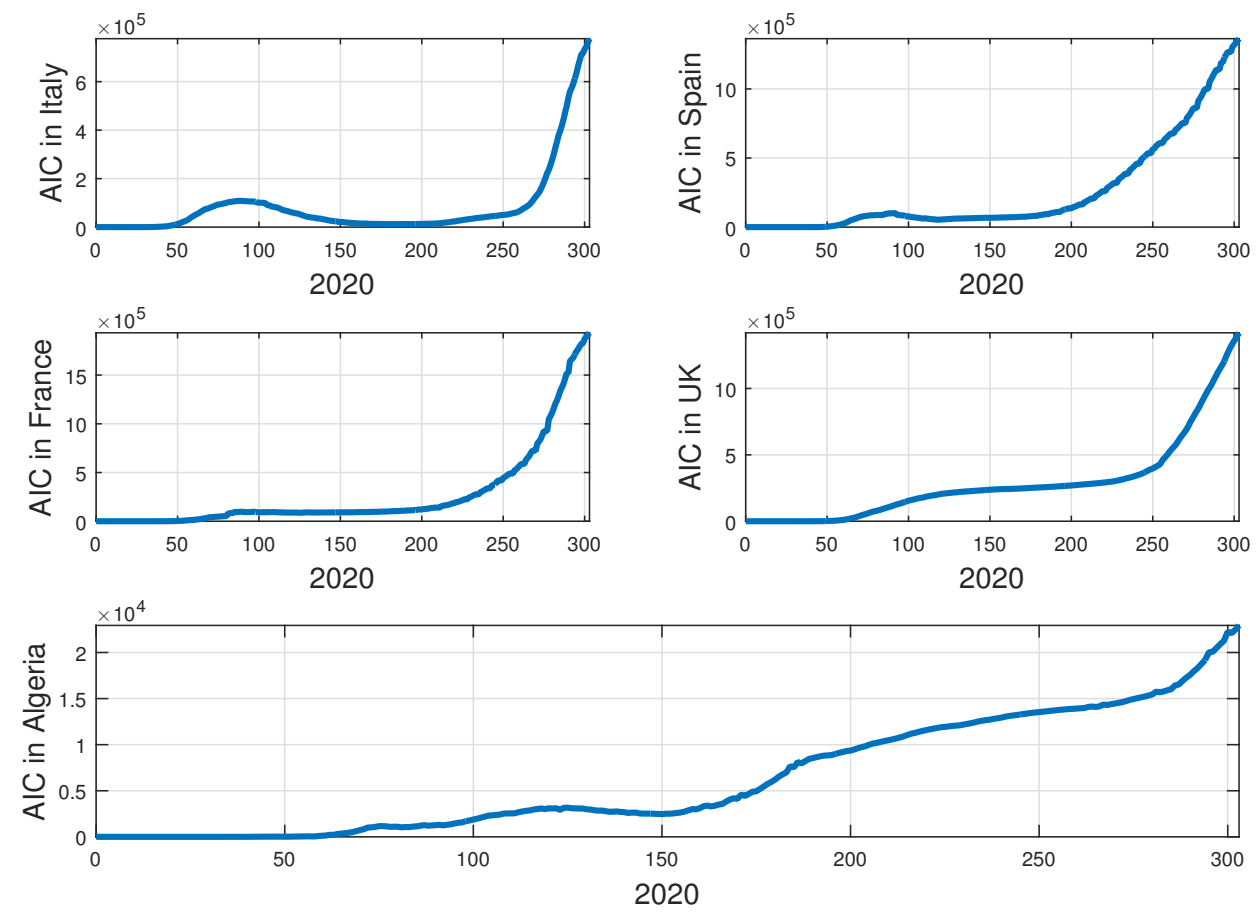

Fig. 10: The evolution of the number of the Active infected cases (DDC) in Italy, Spain, France, UK and Algeria between $t=0(20 / 02 / 2020)$ and $t=304(21 / 11 / 2020)$

part of the death cases is the aged individuals that passed their 60s. This result means that the restriction done by the government helps in reducing the number of infectious diseases. Further, due to the recent release of the social movements, we noticed a huge increase in infectious cases as is been shown in Fig. 10. SO the worst scenario studied in this paper could happen if the situation stays the same.

Sp ain: This country comes just after Italy where it can have more worst scenario than Italy. The basic reproduction number is higher $R_{0}=2.96$. A serious strict public health measures must be taken by the government. Indeed, the peak outbreak epidemic will be reached on 05/05/2020 with 7337600 infection cases. The end of the epidemic will be reached by the beginning of June. It will undertake a painful loss with 100000 death cases. To mention that Spain uses a good lockdown at $t=88$, which led to a reduction of infection cases, this point is the direct application of section 5, but it is hard to use it for a long time. So, the country goes with releasing of the social movements which led to the second increase of infection cases as it is been highlighted in Fig. 10, and the situation must be controlled by the government.

Fr ance: This country is on the same path as Spain. The reproduction number is $R_{0}=3.21$. In [9], it is obtained that $R_{0}=4.45$ (with data before $14 / 03 / 2020$ ) which means that $R_{0}$ decreased (for data before $06 / 04 / 2020$ ). This means that the strict public heal measures are very helpful in reducing the outbreak epidemic of COVID-19. In fact, The peak epidemic outbreak will be reached on $05 / 05 / 2020$ with 3037900 infection cases. The results indicate that this infection approximated to end by the beginning of June. But the lockdown done by the government led to a decrease of infection cases (see Fig. 10) and the second releasing of the movements gives a huge opportunity to the virus to spread (see Fig. 10. So, strict measures in necessary for avoiding the said scenario.

UK: This country is also in a critical state. The basic reproduction number is $R_{0}=3.31$. The peak epidemic spread of the coronavirus will be achieved at 05/05/2020 with 1190000 infection cases. Also, we conclude that the infection will finish by the beginning of June. The approx- 
imation says that this infection will take around 68367 lives mostly the aged individuals if the situation stays the same. The same remark considered for the previous countries can be applied for all countries where the restriction helps in reducing the infection cases and the releasing increases again the number of infectious cases.

Algeria: This country is in the early stage of the spread of COVID19. Indeed, the primary results are not promising, where the basic reproduction number for this country is $R_{0}=$ 3.93 which is dangerously high. the Peak epidemic outbreak will be achieved on 12/05/2020 with 2854400 infection cases. we conclude that the coronavirus epidemic will stay in the Algerian community until the end of June.

Note that these results are true for the case of no measures. In fact that through Fig. 10 we can see that the world witness a second wave and these results can be applied to this second wave. The obtained results show that releasing social movement is very important to keep the balance of the economic system, and this second releasing will lead to a very dangerous increase in infectious cases and the scenario studied in this paper can behold again if the government didn't take a proper measure to stop the spread of this contagious disease.

\section{Conflicts of Interest}

The authors declare no conflict of interest.

\section{Author's Contributions}

All authors carried out the proofs and conceived of the study. All authors read and approved the final manuscript.
7. J. K. Hale, Asymptotic Behavior of Dissipative Systems, Mathematical Surveys and Mono- graphs 25, American Mathematical 48 Society, Providence, RI, 1988

8. Z.Liu, P.Magal, O.Seydi, G.Webb, Understanding Unreported Cases in the COVID-19 Epidemic Outbreak in Wuhan, China, and the Importance of Major Public Health Interventions. Biology 9 (2020) 50.

9. P.Magal, G.Webb, Predicting the number of reported and unreported cases for the COVID-19 epidemic in South Korea, Italy, France and Germany, MedRxiv (2020) doi: https://doi.org/10.1101/2020.03.21.20040154

10. P.Magal, C.C.McCluskey, G.F.Webb, Lyapunov functional and global asymptotic stability for an infection-age model Appl. Anal., 89(7) (2010) 1109-1140

11. R.Porcheddu, C.Serra, D.Kelvin, N.Kelvin, S.Rubino, Similarity in Case Fatality Rates (CFR) of COVID-19/SARS-COV-2 in Italy and China. J Infect Dev Ctries 14 (2020) 125-128. doi: 10.3855/jidc. 12600 .

12. A.Remuzzi, G.Remuzz, COVID-19 and Italy: what next?, Health Policy, (2020) DOI:https://doi.org/10.1016/S01406736(20)30627-9

13. H.Sun, Y.Qiu, H.Yan, Y.Huang, Y.Zhu, S.X.Chen, Tracking and predicting COVID-19 epidemic in China mainland. medRxive 2020, doi: https://doi.org/10.1101/2020.02.17.20024257

14. https://www.who.int/emergencies/diseases/ novel-coronavirus-2019/situation-reports

15. https://en.wikipedia.org/wiki/2020_coronavirus_ outbreak_in_Italy

16. https://en.wikipedia.org/wiki/2020_coronavirus_ outbreak_in_France

17. https://en.wikipedia.org/wiki/2020_coronavirus_ pandemic_in_Spain

18. https://en.wikipedia.org/wiki/2020_coronavirus_ pandemic_in_the_United_Kingdom

19. https://en.wikipedia.org/wiki/2020_coronavirus_ pandemic_in_Algeria

20. https://www.cia.gov/library/publications/ the-world-factbook/fields/341.html

\section{Funding}

This study was funded by the PRFU project No.C00L03UN130120200004, DGRSDT, Algeria.

\section{References}

1. S. Bentout, T.M. Touaoula, Global analysis of an infection age model with a class of nonlinear incidence rates, J.Math. Anal.Appl. 434(2) (2016) 1211-1239.

2. F.Brauer, Z.Shuai, P.van den Driessche, Dynamics of an age of infection cholera model Math. Biosci. Eng., 10(5-6) (2013) 13351349

3. S. Djilali, T.M. Touaoula, S.E.H. Miri, A heroin epidemic model: very general non linear incidence, treat-age, and global stability. Acta Appl. Math. 152(1) (2017) 171-194.

4. S.Djilali, B.Ghanbari, Coronavirus pandemic: A predictive analysis of the peak outbreak epidemic in South Africa, Turkey, and Brazil, Chaos, Solitons and Fractals, 138:109971 (2020).

5. S.Bentout, A.Tridane, S.Djilali, T.M.Touaoula, Agestructured Modeling of COVID-19 Epidemic in the USA, UAE and Algeria, Alexandria Engin. J., (2020) DOI:https://doi.org/10.1016/j.aej.2020.08.053.

6. S.Djilali, L.Benahmadi,A.Tridane, K.Niri, Modeling the Impact of Unreported Cases of the COVID-19 in the North African Countries, Biology 9(11) (2020) DOI: 10.3390/biology9110373 